\title{
Nonlinear Properties of the Rice Statistical Distribution: Theory and Applications in Stochastic Data Analysis
}

\author{
Tatiana Yakovleva \\ Federal Research Center "Computer Science and Control” of the Russian Academy of Sciences, Moscow, Russia \\ Email: tan-ya@bk.ru
}

How to cite this paper: Yakovleva, T. (2019) Nonlinear Properties of the Rice Statistical Distribution: Theory and Applications in Stochastic Data Analysis. Journal of Applied Mathematics and Physics, 7, 2767-2779.

https://doi.org/10.4236/jamp.2019.711190

Received: October 8, 2019

Accepted: November 10, 2019

Published: November 13, 2019

\begin{abstract}
The paper considers the theoretical basics and the specific mathematical techniques having been developed for solving the tasks of the stochastic data analysis within the Rice statistical model in which the output signal's amplitude is composed as a sum of the sought-for initial value and a random Gaussian noise. The Rician signal's characteristics such as the average value and the noise dispersion have been shown to depend upon the Rice distribution's parameters nonlinearly what has become a prerequisite for the development of a new approach to the stochastic Rician data analysis implying the joint signal and noise accurate evaluation. The joint computing of the Rice distribution's parameters allows efficient reconstruction of the signal's informative component against the noise background. A meaningful advantage of the proposed approach consists in the absence of restrictions connected with any a priori suppositions inherent to the traditional techniques. The results of the numerical experiments are provided confirming the efficiency of the elaborated approach to stochastic data analysis within the Rice statistical model.
\end{abstract}

\section{Keywords}

Stochastic Data, Rice Distribution, Probability Density Function, Maximum Likelihood Technique, Method of Moments, Noise Dispersion

\section{Introduction}

The techniques of the stochastic data analysis are obviously based upon and determined by the statistical properties of the data to be analyzed. The Rice statistical distribution has recently become a subject of increasing scientific interest because of a wide circle of stochastic data processing tasks which are adequately 
described just by the Rice statistical model [1]. In particular, this circle includes the tasks of high precision measurements in the systems of the magnetic-resonance visualization, at radar signals' analysis, in the field of optical metrology, at distance measurements, in ranging systems, at determining the object's geometrical parameters, at non-destructive control, etc. [2] [3] [4] [5].

The Rice distribution describes an information processing problems in which an initially determined signal is distorted by an inevitable random noise generated by many independent normally distributed summands of zero mean value, i.e. the output signal is composed as a sum of the sought-for initial signal and the Gaussian noise component. The variable to be measured and analyzed is an amplitude, or an envelope of the resulting signal, which is known to obey the Rice statistical distribution [1]. The so-called two-parameter approach to the Rician signals' analysis consists in solving the task of joint determination of the both parameters of the Rice distribution. In contrast to the traditional one-parameter approximation this approach is free of limitations that are inherent to the one-parametric approximation based upon the supposition that one of the task statistical parameters-the noise dispersion-is known a priori [2] [3]. That's why the technique of the two-parametric task solution ensures much more correct estimation of the required values.

The paper provides both the theoretical basics of the elaborated approach based upon the Rician signals' two-parameter analysis and some results of its computer simulation.

\section{Theoretical Basics of the Rician Data Analysis}

The Rician distribution is known to describe an amplitude of the random value, formed by summing an initially determined complex signal and the Gaussian noise distorting this signal. By virtue of the central limit theorem, this situation is rather a typical one at describing various physical processes.

Let $A$ be a determined value that characterizes the physical process to be considered. This value is inevitably distorted by the Gaussian noise created by a great number of independent noise components, while the measured and analyzed value is an amplitude, or the envelope of the resulting signal. The Gaussian noise distorting the initial determined signal is characterized by a zero mean value and a dispersion $\sigma^{2}$. Thus the task consists in the analysis of the signal's amplitude $x=\sqrt{x_{\mathrm{Re}}^{2}+x_{\mathrm{Im}}^{2}}$.

The real $x_{\mathrm{Re}}$ and imaginary $x_{\mathrm{Im}}$ parts of the complex signal with amplitude $x$ are random Gaussian values with the mathematical expectations $\overline{x_{\mathrm{Re}}}$ and $\overline{x_{\mathrm{Im}}}$, satisfying the condition ${\overline{x_{\mathrm{Re}}}}^{2}+{\overline{x_{\mathrm{Im}}}}^{2}=A^{2}$, and dispersion $\sigma^{2}$. The random value $x$ is known to obey the Rice distribution with parameters $v=A$ and $\sigma^{2}$ [1]. Obviously, the value of $x$ belongs to the subset of the not-negative real numbers: $x \in(0, \infty)$. The ratio of the Rician parameters $S N R=v / \sigma$ characterizes the signal-to-noise ratio.

In other words, the Rician random variable $x$ represents the amplitude of the 
signal with the Gaussian real and imaginary parts.

The Rician probability density function is given by the following formula:

$$
P\left(x \mid v, \sigma^{2}\right)=\frac{x}{\sigma^{2}} \cdot \exp \left(-\frac{x^{2}+v^{2}}{2 \sigma^{2}}\right) \cdot I_{0}\left(\frac{x v}{\sigma^{2}}\right),
$$

where $I_{0}$ is the modified Bessel function of the first kind of order zero [6].

Here and below we'll use the following denotations: $I_{\alpha}(z)$ is the modified Bessel function of the first kind (or the Infeld function) of the order $\alpha ; x_{i}$ is the signal's value measured as the $i$-th element of a sample; $n$ is the quantity of elements in a sample, called also a sample's length.

The final purpose of the Rician data processing is evidently the evaluation of value $A$ that characterizes the process under the study and coincides with parameter $v$ of the Rice distribution.

Let's denote the various types of the signal's averaging by the angular brackets: $\left\langle x^{k}\right\rangle=\frac{1}{n} \sum_{i=1}^{n} x_{i}^{k}$, while the average value at infinite large sample's length is denoted by the over-bar: $\overline{x^{k}}=\lim _{n \rightarrow \infty} \frac{1}{n} \sum_{i=1}^{n} x_{i}^{k}$.

The Rician value's mathematical expectation and dispersion are known to be expressed by the following formulas [7]:

$$
\begin{gathered}
\bar{x}=\sigma \cdot \sqrt{\pi / 2} \cdot L_{1 / 2}\left(-v^{2} / 2 \sigma^{2}\right), \\
\sigma_{x}^{2}=\overline{x^{2}}-\bar{x}^{2}=2 \cdot \sigma^{2}+v^{2}-\sigma^{2} \cdot \frac{\pi}{2} \cdot L_{1 / 2}^{2}\left(-v^{2} / 2 \sigma^{2}\right),
\end{gathered}
$$

where function $L_{1 / 2}(z)$ is the Laguerre polynomial.

From the mathematical peculiarities of the Rician distribution noticed above it follows that the Rician signal's mean value (2) and its dispersion (3) depend on the both Rician parameters: $v$ and $\sigma^{2}$ and this dependence is essentially nonlinear. This means that the task of reconstructing the initial, un-noised signal's value $v$ at a priori unknown Gaussian noise dispersion $\sigma^{2}$ can be solved only on the basis of joint determination of both parameters $v$ and $\sigma^{2}$.

The both Rician parameters have a certain physical sense, namely: $\sigma^{2}$ is $s$ dispersion of the Gaussian noise distorting the initial signal while parameter $v$ coincides with the value of the initial determined signal $v=A$. That's why the task of the calculation of the both Rician statistical parameters is of special importance at data processing as it is directly connected with solving the problem of the separation of the informative and the noise components of the analyzed signal. In other words, an efficient reconstruction of the Rician signal against the noise demands solving the two-parameter task, or the task of the joint evaluation of the both a priori unknown parameters $v$ and $\sigma^{2}$.

The essence of the conception of two-parameter analysis of the Rician distributed stochastic data consists in understanding the necessity of such a joint evaluation of both unknown Rician parameters $v$ and $\sigma$ of the analyzed signal. An important distinctive feature of the considered task formulation is the 
absence of any a priori suppositions and restrictions concerning the values of the parameters $v$ and $\sigma$ : the task is being solved only on the basis of the sampling measured data analysis.

The Rice distribution is known to be mutually connected with two other statistical distributions: the Rayleigh distribution and the Gauss distribution. Namely, it is known that the Rice distribution is to be transformed into the Gauss distribution at increasing the value of the signal-to-noise ratio, being determined by the Rician parameters' ratio: $S N R=v / \sigma$, while in opposite limiting case of negligibly small value of the signal-to-noise ratio the Rice distribution is transformed into the Rayleigh distribution.

By virtue of the specifics of the Rice distribution the analysis of the Rician data demands the development of special methods and the corresponding mathematical apparatus.

\section{Nonlinearity of the Rician Value Characteristics}

It is well known that at the Gaussian data analysis a traditional and efficient filtration tool is the data averaging. However, in contrast to the case of the Gaussian distribution the average value of the Rician signal $\bar{x}$ does not coincide with the value of the sought for useful signal $v$, as it follows from (2). This is illustrated by Figure 1 where the average value of the Rician signal $\bar{x}$ is depicted by the curve line, while the value of the sought for useful signal $v$ is depicted by the straight line outgoing from the origin of coordinates. The plots in Figure 1 correspond to the constant value of parameter $\sigma: \sigma=1$, so that the points of the abscissa axes correspond to the value of signal-to-noise ratio $S N R=v / \sigma$.

Therefore if one applies to the Rician data processing the traditional technique of the filtration by means of the data averaging, then within the range of small values of the signal-to-noise ratio the result will be just a leveling of the real signal values.

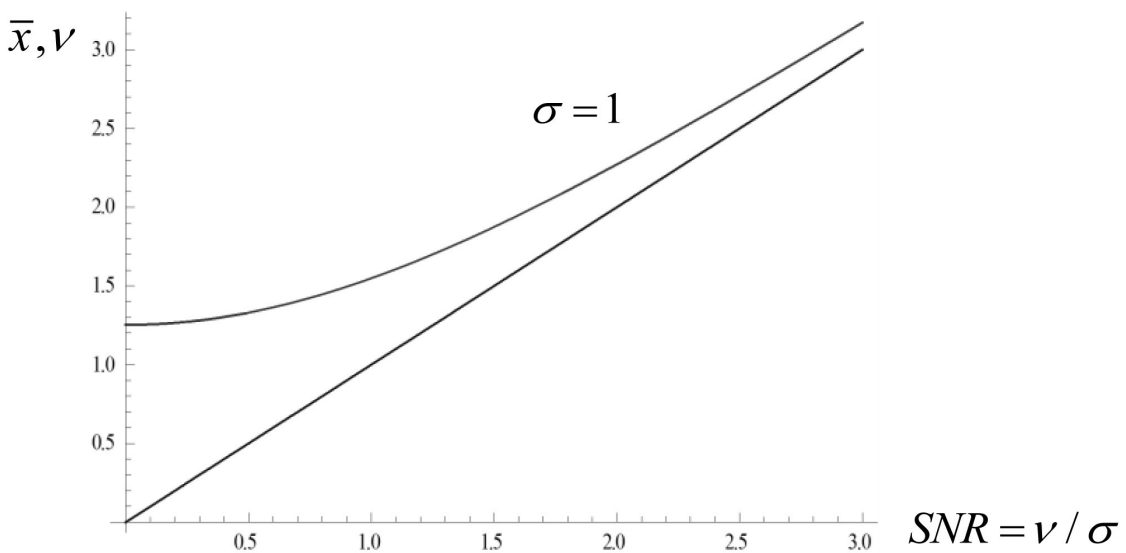

Figure 1. The illustration of the divergency of the Rician signal's averaged value $\bar{x}$ and Rician parameter $v$ as dependent on the signal-to-noise ratio $S N R=v / \sigma$. 
Let us consider the interconnection between the Rician signal's dispersion value and the dispersion of the Gaussian noise forming the Rician signal. As mentioned above, the average value of the Rician signal does not coincide with the initial, un-noised signal. Similarly the dispersion of the Rician signal does not coincide with the dispersion of Gaussian noise forming the Rician signal from the initially determined value. These properties are inherent to the Rice distribution and, in contrast to the Gaussian statistical model, do not allow analyzing data just by simple averaging. Instead the indicated peculiarities of the Rician random variable predetermine the necessity of the development of special theoretical approach for the Rician data analysis and processing.

Taking into account the known properties of the Laguerre polynomial [6], namely: $\lim _{z \rightarrow 0} L_{1 / 2}(z)=1$, from the expression (2) for the Rician variable dispersion $\sigma_{x}^{2}=\overline{x^{2}}-\bar{x}$ in the limiting case $v / \sigma \ll 1$, when the value of the useful signal is much less than the Gaussian noise value, we get

$$
\sigma_{x}^{2} \underset{v / \sigma \ll 1}{\rightarrow} \sigma^{2} \cdot\left(2-\frac{\pi}{2}\right)
$$

Formula (4) coincides with the known formula for the random variable with the Rayleigh distribution, what is not unexpected in virtue of the interconnection between the Rice and the Rayleigh distributions: at $v / \sigma \ll 1$ the Rice distribution is being transformed into the Rayleigh distribution.

In another limiting case, when the value of the useful signal significantly exceeds the noise level, i.e. at $v / \sigma \gg 1$, taking into account the known representation of the Laguerre polynomial through the modified Bessel functions as well as the asymptotic representation of the modified Bessel functions [6] from (3) we get: $\sigma_{x}^{2} \underset{v / \sigma \rightarrow \infty}{\rightarrow} \sigma^{2}$. So in a limiting case of very high signal-to-noise ratio the Rician signal's dispersion coincides with the Gaussian noise dispersion what is natural taking into account the interconnection of the Rician and the Gaussian distributions: at $v / \sigma \gg 1$ the Rician distribution is being transformed into the Gaussian distribution with the corresponding values of parameters $v$ and $\sigma^{2}$.

These theoretical results are illustrated in Figure 2: the graph in Figure 2(a) presents the Rician signal dispersion, calculated by Formula (3) as depending upon the signal-to-noise ratio $S N R=v / \sigma$ at constant value of the Gaussian noise dispersion $\sigma=1$ (dashed straight line). In Figure 2(b) there is presented the Rician signal dispersion measured in a numerical experiment as dependent on parameter $v$. The sampled data were obtained for each signal value $v$ by means of the Rician random numbers' generator. At the numerical experiment the results of which are presented in Figure 2(b), parameter $v$ was changing within the range from 0 to 10 with the step 0.05 , while the sample length was $n=256$. Based upon the sampled Rician data there was calculated the Rician signal dispersion depicted by the solid fluctuating line. The dashed horizontal line corresponds to the initially designated value of the Rician parameter $\sigma=1$, being determined by the Gaussian noise dispersion. 


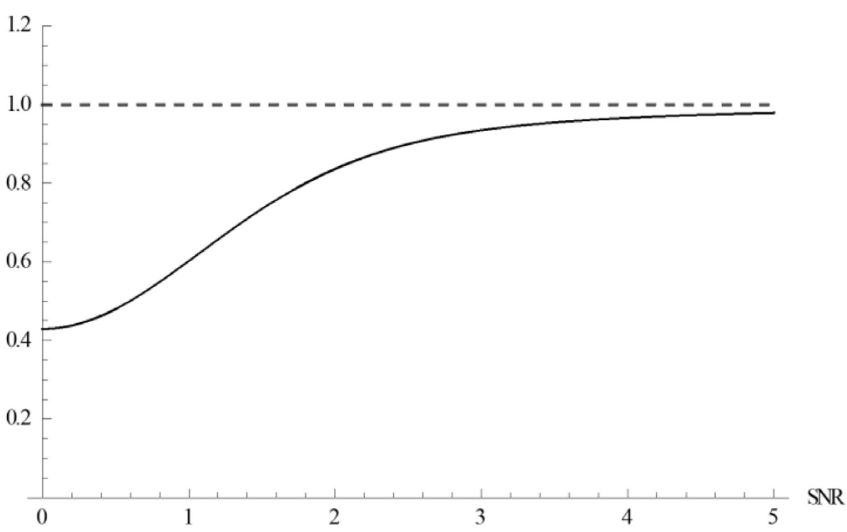

(a)

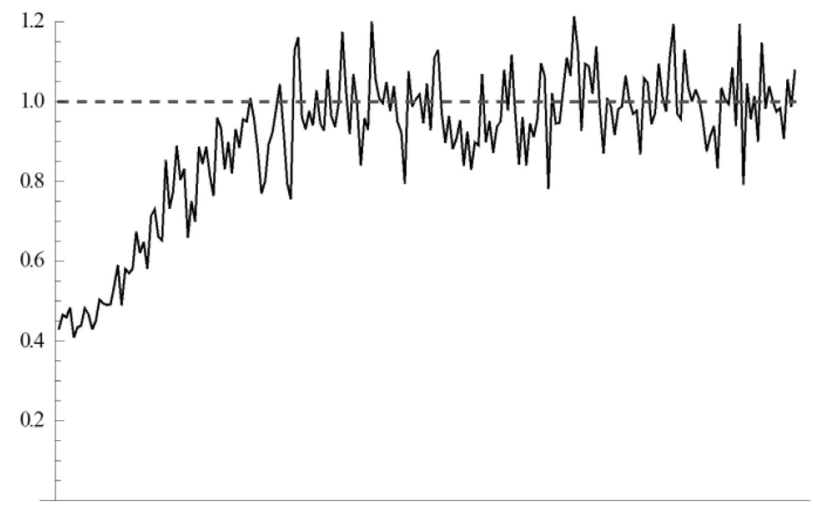

(b)

Figure 2. The graphs of the Rician signal's dispersion calculated by Formula (3) (a) and measured in numerical experiment (b).

The numerical experiment's results presented in Figure 2(b) accord with Formula (3) for the Rician variable dispersion depicted in Figure 2(a).

The above indicated nonlinearities of the Rice distribution are such that the reconstruction of the initial, un-noised signal against the noise background is possible only by means of estimation of the both Rician parameters, or, in other words, by solving the two-parameter task.

\section{The Principle Techniques and Equations of the Two-Parameter Approach to Rician Data Analysis}

The particular theoretical methods having been developed within the two-parameter analysis of the Rician signal in [8] [9] [10] [11] are based upon the underlying principles of mathematical statistics.

Before turning to the detailed consideration of a mathematical technique the present paper deals with let us provide the brief information about the two-parameter techniques of Rician data analysis elaborated earlier in [8] [9] [10] [11]. These are the following specific techniques: the method of moments based on the measured data for the random value's 1-st and 2-nd moments, des- 
ignated as MM12; the method of moments based on measurements of the 2-nd and the 4-th moments, designated as MM12; the two-parametric maximum likelihood method, designated as ML; the method based upon processing of sample measurements of the 1-st and the 3-rd raw moments of the random Rician data, designated as MM13. Each of these methods is based on solving the corresponding nonlinear equations' system for the sought for parameters $v$ and $\sigma^{2}$. Below there are provided the systems of equations corresponding to these methods [8] [9] [10] [11].

The system of equation for method MM12 looks as follows:

$$
\left\{\begin{array}{l}
\sigma \cdot \sqrt{\pi / 2} \cdot e^{-\frac{v^{2}}{4 \sigma^{2}}}\left[\left(1+\frac{v^{2}}{2 \sigma^{2}}\right) I_{0}\left(\frac{v^{2}}{4 \sigma^{2}}\right)+\frac{v^{2}}{2 \sigma^{2}} I_{1}\left(\frac{v^{2}}{4 \sigma^{2}}\right)\right]=\bar{x}, \\
2 \sigma^{2}+v^{2}=\overline{x^{2}}
\end{array}\right.
$$

Method MM24 is rather an original and simple in its realization with equations' system for method MM24 as:

$$
\left\{\begin{array}{l}
\overline{x^{2}}=2 \cdot \sigma^{2}+v^{2} \\
\overline{x^{4}}=8 \cdot \sigma^{4}+8 \cdot \sigma^{2} \cdot v^{2}+v^{4}
\end{array}\right.
$$

For ML method we have the following system of equations:

$$
\left\{\begin{array}{l}
v=\frac{1}{n} \sum_{i=1}^{n} x_{i} \cdot I_{1}\left(x_{i} \cdot v / \sigma^{2}\right) / I_{0}\left(x_{i} \cdot v / \sigma^{2}\right) \\
\sigma^{2}=\left(\left\langle x^{2}\right\rangle-v^{2}\right) / 2 .
\end{array}\right.
$$

The system of equation for method MM13 is as follows:

$$
\left\{\begin{array}{l}
\langle x\rangle=\sigma \cdot \sqrt{\pi / 2} \cdot{ }_{1} F_{1}\left(-\frac{1}{2} ; 1 ;-\frac{v^{2}}{2 \sigma^{2}}\right) \\
\left\langle x^{3}\right\rangle=3 \cdot \sigma^{3} \cdot \sqrt{\pi / 2} \cdot{ }_{1} F_{1}\left(-\frac{3}{2} ; 1 ;-\frac{v^{2}}{2 \sigma^{2}}\right) .
\end{array}\right.
$$

A mathematical technique to be considered in detail in the present paper method is based upon combining the maximum likelihood technique and the method of moments. Let us designate as MLM. To get the equations for this technique let us consider a sample of $n$ measurements of the Rician value $x$. Suppose that the $i$-th measurement in the sample results in $x_{i} \quad(i=1, \ldots, n)$. Then the joint probability density function for this event is equal to the product of the probability density functions for each measurement in the sample:

$$
L\left(v, \sigma^{2}\right)=\prod_{i=1}^{n} P\left(x_{i} \mid v, \sigma^{2}\right)
$$

where function $P\left(x_{i} \mid v, \sigma^{2}\right)$ is determined by expression (1). Function $L\left(v, \sigma^{2}\right)$ is a likelihood function and at the obtained results of the sample measurements it is defined by Rician statistical parameters $v$ and $\sigma^{2}$. The maximum likelihood technique is known to consist in finding those values of parameters $v$ and $\sigma^{2}$ which maximize the likelihood function $L\left(v, \sigma^{2}\right)$, or, 
equivalently, its logarithm:

$$
\begin{aligned}
& \ln L\left(v, \sigma^{2}\right)=\sum_{i=1}^{n} \ln P\left(x_{i} \mid v, \sigma^{2}\right) \\
& =\sum_{i=1}^{n}\left\{\ln x_{i}-\ln \sigma^{2}-\frac{x_{i}^{2}+v^{2}}{2 \cdot \sigma^{2}}+\ln I_{0}\left(\frac{x_{i} v}{\sigma^{2}}\right)\right\} .
\end{aligned}
$$

As the first equation for method MLM we'll use the maximum likelihood equation having been obtained by equalization to zero of the partial derivative of logarithmic likelihood function (9) by parameter $v: \frac{\partial}{\partial v} \ln L\left(v, \sigma^{2}\right)=0$. Taking into account (3) results in:

$$
\sum_{i=1}^{n} \frac{\partial}{\partial v} \ln I_{0}\left(\frac{x_{i} v}{\sigma^{2}}\right)-\frac{n \cdot v}{\sigma^{2}}=0
$$

Having used the known expression $\frac{d}{d z} I_{0}(z)=I_{1}(z) \quad$ ([6]), we get from (10):

$$
\frac{1}{\sigma^{2}} \sum_{i=1}^{n} x_{i} \cdot \frac{I_{1}\left(\frac{x_{i} v}{\sigma^{2}}\right)}{I_{0}\left(\frac{x_{i} v}{\sigma^{2}}\right)}-\frac{n \cdot v}{\sigma^{2}}=0
$$

As the second equation for method MLM under consideration we'll use Formula (2) for the first moment of Rician random value [7]. Then we get the following system of equations for calculation of parameters $v$ and $\sigma^{2}$ by method MLM:

$$
\left\{\begin{array}{l}
v=\frac{1}{n} \sum_{i=1}^{n} x_{i} \cdot \tilde{I}\left(\frac{x_{i} \cdot v}{\sigma^{2}}\right) \\
\frac{1}{n} \sum_{i=1}^{n} x_{i}=\sigma \sqrt{\frac{\pi}{2}} \cdot L_{1 / 2}\left(-\frac{v^{2}}{2 \sigma^{2}}\right)
\end{array}\right.
$$

In the first equation of system (12) the denotation $\tilde{I}$ is used for the function $\tilde{I}(z)=\frac{I_{1}(z)}{I_{0}(z)}$ which is equal to the ratio of modified Bessel functions of the first type of the first and the zeroth orders. The properties of function $\tilde{I}$ have been investigated in detail in [11]: this function is smooth, monotonically increasing, up-convex and asymptotically tends to infinity.

An important theoretical result consists in the fact that for each above mentioned two-parameter methods the corresponding system of two nonlinear equations for two variables $v$ and $\sigma^{2}$ can be reduced to one equation for just one variable. This allows an essential decreasing of the computational resources needed for the task solving.

Below as an example we'll consider in more detail and provide the final formulas and the numerical testing results for one of the above mentioned techniques, namely-for the combined method MLM.

Equations' system (12) is an essentially nonlinear system for two unknown parameters: $v$ и $\sigma^{2}$. Let us introduce a new variable: 


$$
r=\frac{v^{2}}{2 \sigma^{2}}
$$

This variable characterizes the value of the signal-to-noise ratio. Then from (12) we'll get the following equations' system for a pair variables $(r, \sigma)$ (instead of a pair $(v, \sigma))$ :

$$
\left\{\begin{array}{l}
\sigma \cdot \sqrt{2 r}=\frac{1}{n} \sum_{i=1}^{n} x_{i} \cdot \tilde{I}\left(\frac{x_{i}}{\sigma} \sqrt{2 r}\right) \\
\langle x\rangle=\sigma \cdot \sqrt{\frac{\pi}{2}} \cdot L_{1 / 2}(-r)
\end{array}\right.
$$

In (14) $\langle x\rangle=\frac{1}{n} \sum_{i=1}^{n} x_{i}$ is a measured in samples value of the first moment, or an average magnitude of Rician value $x$. From the second equation of (14) we get for variable $\sigma$ a following expression:

$$
\sigma=\frac{\langle x\rangle}{\sqrt{\pi / 2} \cdot L_{1 / 2}(-r)}
$$

Substituting (15) into the first equation of system (14) one can get the following equation for variable $r$.

$$
2 \sqrt{r / \pi} \cdot \frac{\langle x\rangle}{L_{1 / 2}(-r)}=\frac{1}{n} \sum_{i=1}^{n} x_{i} \tilde{I}\left(\frac{x_{i} \sqrt{\pi \cdot r}}{\langle x\rangle} L_{1 / 2}(-r)\right)
$$

Therefore, the task of solving system (14) of two equations for two unknown variables $(r, \sigma)$ has been reduced to the task of solving one Equation (16) for just one unknown variable $r$.

So, the calculation of the required value of the useful signal $v$ and the noise dispersion $\sigma^{2}$ by technique MLM consists in the following: by substituting the sampled data for the measured stochastic signal's value $x_{i}, i=1, \ldots, n$ into Equation (16) one can find a solution of this equation for variable $r$ and then calculate the noise parameter $\sigma$ be means of Formula (15). Then, using the obtained magnitudes for $\sigma$ and $r$ one can get from (13) a required parameter of useful signal's value: $v=\sqrt{2 \sigma^{2} r}$.

\section{Results of Numerical Simulation}

This section presents some results of the computer simulation of solving the task of the Rician signal and noise parameters evolution at data analysis by means of the above mathematical technique MLM based upon combining the maximum likelihood technique and the method of moments.

At conducting the numerical experiments the data obeying the Rice statistical distribution have been generated on two-dimensional grid with the nodes corresponding to various initial values of the signal and the noise parameters. By using the sampled values of Rician signal in each node of the grid the required values of the signal and noise parameters have been calculated by elaborated algorithm, i.e. by means of solving Equations (15)-(16). 
Figure 3 presents the graphs for the signal parameter $v$ (Figure 3(a)) and noise parameter $\sigma$ (Figure 3(b)) having been calculated at various values of the signal-to-noise ratio.

Figure 3(a) shows the dependencies of the deviations of the numerically calculated values of parameter $v$ from its real, initially specified value of this parameter at various values of parameter $\sigma$. The points along the abscissa axis correspond to grid nodes for initially specified values of parameter $v$, while the points along the ordinate axis correspond to the calculated values of this parameter. Thereby, the deviations of the broken solid, dashed and dot-dashed lines

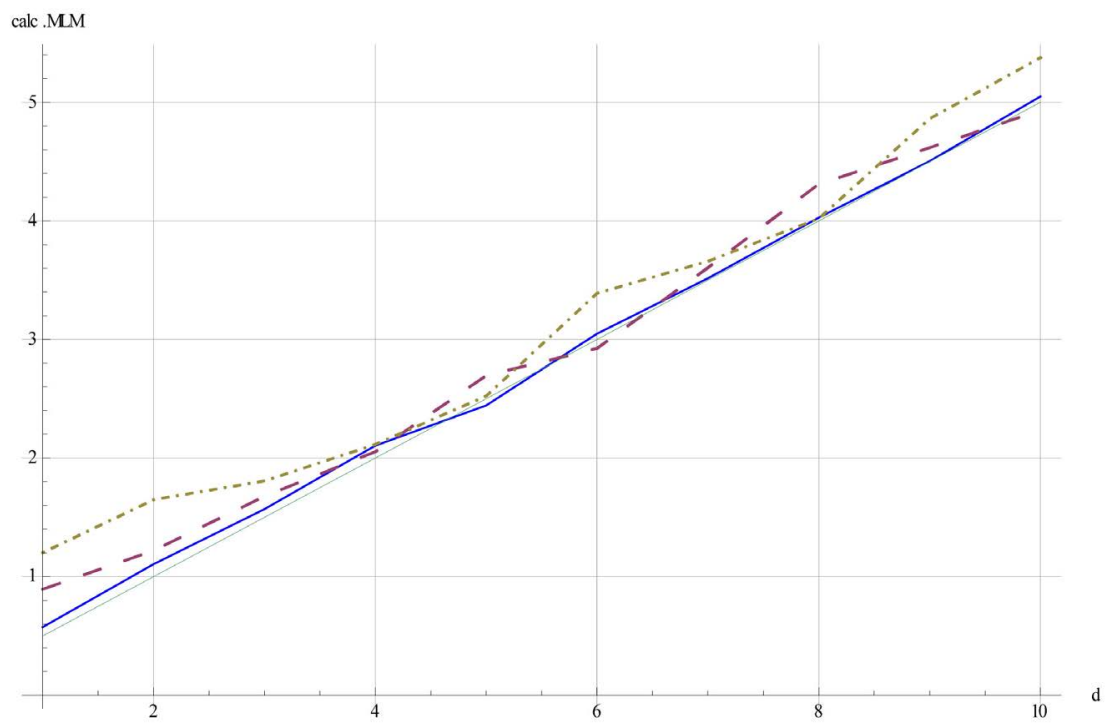

(a)

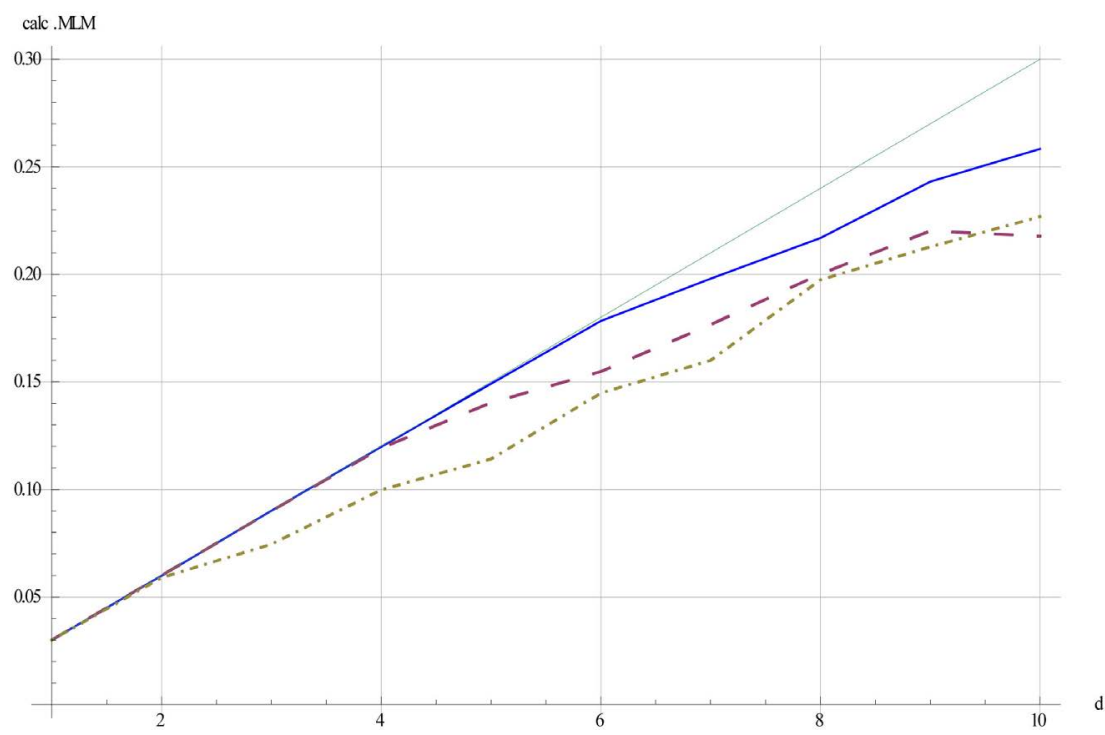

(b)

Figure 3. The results of the Rician parameters computing. (a) The results of numerical calculation of the signal value $v$ at various magnitudes of noise parameter $\sigma$; (b) The results of the numerical calculation of the noise parameter $\sigma$ at various magnitudes of the signal parameter $v$. 
from the straight line characterize the accuracy of the calculations. At the simulation the initial values of parameter $v$ were changed within the range from 0.5 to 5.0 with calculation step 0.5 , and parameter $\sigma$-in the range from 0.5 to 1.5 with step 0.1 . The sample length $n$, i.e. the number of measurements in a sample, in the presented variants of numerical experiments was $n=4$, while the results of calculation were averaged by 25 samples (in real systems of digital signal processing the number of averages samples is usually equal to $10^{3} \div 10^{4}$ ). In Figure 3(a) the solid, dashed and dot-dashed lines illustrated the accuracy of calculation of the signal parameter $v$ at the following specified values of parameter $\sigma$ : $\sigma=0.5$ (solid line); $\sigma=1.0$ (dashed line); $\sigma=1.5$ (dot-dashed line). The graphs demonstrate the expected dependence of the calculations' accuracy on the value of the signal-to-noise ratio: while the initially specified magnitude of noise parameter $\sigma$ is decreasing, i.e. while the signal-to-noise ratio is increasing, the accuracy of the numerical calculation the signal parameter $v$ is noticeably growing.

Figure 3(b) illustrates the results of the numerical calculation of the noise parameter $\sigma$ by the above technique at various initially specified values of useful signal $v$. The shown graphs demonstrate the deviations of calculated values of parameter $\sigma$ from the real values of this parameter depicted by a straight line. The points along the abscissa axis on the both graphs correspond to the reference points of initially given values of parameter $\sigma$, and along the ordinate axis-to the calculated values of this parameter, i.e. the deviations of the broken solid, dashed and dot-dashed lines from the straight line characterize the accuracy of the calculations. In Figure 3(b) the solid, dashed and dot-dashed lines have been obtained for initially specified values of parameter $v: v=3.0$, $v=2.0$ and $v=1.0$, correspondingly. As in the case of the signal parameter calculation, the accuracy of computing the noise parameter $\sigma$ essentially depends on the value of the signal-to-noise ratio.

The presented graphical data illustrate rather a high efficiency of the elaborated technique for the joint signal and noise parameters' calculation.

\section{Conclusion}

The paper considers an approach to solving the task of the stochastic data analysis within the conditions of the Rice statistical distribution and presents the results of the theoretical study of this distribution's peculiarities. The nonlinear character of the Rician value's average and dispersion as dependent of the Rician parameters have been shown to cause the necessity of the special mathematical apparatus development for the Rician stochastic data analysis and processing. The so-called two-parameter approach to solving this task, based upon the joint estimation of the both Rician parameters, has been considered and a few specific mathematical techniques having been developed within this approach are presented. The paper provides the theoretical elaboration of the technique based upon combining the maximum likelihood method and the method of moments. 
The task of solving a system two nonlinear equations for two unknown variables has been mathematically reduced to solving just one equation for one unknown variable, what essentially decreases the necessary calculating resources. The provided results of the numerical experiments confirm the efficiency of the developed technique for solving the problem of the stochastic Rician data analysis ensuring a high precision of the signal and noise parameters estimation.

\section{Acknowledgements}

The work has been supported by RFBR, project N17-07-00064 within the Fundamental Research program.

\section{Conflicts of Interest}

The author declares no conflicts of interest regarding the publication of this paper.

\section{References}

[1] Rice, S.O. (1944) Mathematical Analysis of Random Noise. Bell Syst. Tech. Journal, 23, 282-322. https://doi.org/10.1002/j.1538-7305.1944.tb00874.x

[2] Benedict, T.R. and Soong, T.T. (1967) The Joint Estimation of Signal and Noise from the Sum Envelope. IEEE Trans. Inf. Theory, IT-13, 447-454. https://doi.org/10.1109/TIT.1967.1054037

[3] Talukdar, K.K. and Lawing, W.D. (1991) Estimation of the Parameters of Rice Distribution. J. Acoust. Soc. Amer., 89, 1193-1197. https://doi.org/10.1121/1.400532

[4] Sijbers, J., den Dekker, A.J., Scheunders, P. and Van Dyck, D. (1998) Maximum-Likelihood Estimation of Rician Distribution Parameters. IEEE Transactions on Medical Imaging, 17, 357-361. https://doi.org/10.1109/42.712125

[5] Carobbi, C.F.M. and Cati, M. (2008) The Absolute Maximum of the Likelihood Function of the Rice Distribution: Existence and Uniqueness. IEEE Trans. on Instrumentation and Measurement, 57, 682-689. https://doi.org/10.1109/TIM.2007.913823

[6] Abramovits, M. and Stegun, I. (1979) Handbook of Mathematical Functions. Nauka.

[7] Park Jr., J.H. (1961) Moments of Generalized Rayleigh Distribution. Q. Appl. Math., 19, 45-49. https://doi.org/10.1090/qam/119222

[8] Yakovleva, T.V. and Kulberg, N.S. (2014) Special Features of the Likelihood Function of the Rice Statistical Distribution. Doklady Mathematics, 90, 472-475. https://doi.org/10.1134/S1064562414050159

[9] Yakovleva, T.V. (2014) Conditions of Rice Statistical Model Applicability and Estimation of the Rician Signal's Parameters by Maximum Likelihood Technique. Computer Research and Modeling, 6, 13-25. https://doi.org/10.20537/2076-7633-2014-6-1-13-25

[10] Yakovleva, T.V. and Kulberg, N.S. (2014) Mathematical Statistics Methods as a Tool of Two-Parameter Magnetic-Resonance Image Analysis. Informatics and its Application, 8, 79-89.

[11] Yakovleva, T.V. and Kulberg, N.S. (2013) Noise and Signal Estimation in MRI: 
Two-Parametric Analysis of Rice-Distributed Data by Means of the Maximum Likelihood Approach. American Journal of Theoretical and Applied Statistics, 2, 67-79. https://doi.org/10.11648/j.ajtas.20130203.15 